Portland State University

PDXScholar

$11-11-2018$

\title{
The Effects of Organic and Conventional Agriculture on Bee Populations in the United States
}

Jordan Bicknell

Portland State University

Follow this and additional works at: https://pdxscholar.library.pdx.edu/honorstheses

Let us know how access to this document benefits you.

\section{Recommended Citation}

Bicknell, Jordan, "The Effects of Organic and Conventional Agriculture on Bee Populations in the United States" (2018). University Honors Theses. Paper 648.

https://doi.org/10.15760/honors.663

This Thesis is brought to you for free and open access. It has been accepted for inclusion in University Honors Theses by an authorized administrator of PDXScholar. Please contact us if we can make this document more accessible: pdxscholar@pdx.edu. 


\title{
The Effects of Organic and Conventional Agriculture on Bee Populations in the United States
}

\author{
By \\ Jordan Bicknell
}

An Undergraduate Honors Thesis Submitted in Partial Fulfillment of the Requirements for the Degree of

Bachelor of Science

In

University Honors

And

Environmental Science and Management

Thesis Advisor

Megan Horst

Portland State University

2018 


\section{Introduction}

Conventional agriculture is known to cause to a multitude of environmental problems.

Eutrophication, sedimentation and reduced water quality are all impacts being addressed by environmental scientists from agricultural activity. This paper focuses on environmental impacts of agriculture that affect bee populations. Farming is dependent on pollinators, particularly bees, to produce fruit, vegetables and other crops for human consumption but conventional farming practices have caused habitat destruction and reduced food availability, subsequently elevating stresses faced by bees. This is occurring in conjunction with a rising demand for food. Pollinator stress and increased demands by the agricultural industry can lead to degradation of the natural environment and the extinction of bees. Based on the stresses presented by conventional agriculture, it appears that the converting farmland to adopt organic practices will serve as a logical avenue to mitigate these stresses on bees. This paper shows that in order for organic farming to provide significant benefits, conversion would have to occur under highly specific circumstances.

Colony Collapse Disorder, or CCD, has been widely reported over the past few years in managed honey bee colonies on farms in both the United States and Europe and the term "pollinator crisis" was introduced to the world as a result. Among other propositions, the global expansion of the conventional agricultural industry has been proposed as a prominent source for the declines seen in managed honey bees but wild/native populations of bees also face threats posed by conventional farming. Conventional farming is characterized by a few critical operations, all acknowledged throughout environmental discourse communities to negatively impact bees and the environment. Growing the same crop over a large area of land (i.e. monocropping) reduces natural habitat and removes food resources and is a defining feature of conventional farms. Additionally, using synthetic and chemical-based pest control to accommodate the large number of crops causes mortality and spreads disease. The United States Department of Agriculture (USDA) Organic Certification was an effort made to mitigate the negative impacts of conventional agriculture and promote environmental stewardship.

Current USDA Organic certified farms can benefit bees as they are banned from using synthetic fertilizers and pesticides and must provide habitat between fields and around the farm. Bees have access to more resources that can assist in maintaining healthy populations and potentially provide enough to allow for population growth. In recent years, organic farming has been growing in response to the increased awareness of the environmental risks posed by conventional farming in an effort to shift the paradigm of food production away from traditional practices. The combination of the bee declines and increased prevalence of organic farming prompt the investigation of whether or not organic farming practices are 1) beneficial to bee species 2) would assist in preventing further population declines and risks to bees and 3) could be a restoration tool to revive declining bee populations. Bees are the focus of this literature review as they occupy much of the current literature and facilitate the spread of this information as bees and the concern for them overlaps both the scientific and public realms. This paper reviews current literature on the effects of organic farming on bee populations in comparison to 
conventional farming. The aim of this paper is to assess the efficacy of organic farm management as a solution for, 1) mitigating risks faced by bees from conventional farming practices and 2) restoring bee populations and species that have faced declines. Results indicate that converting to organic farming is not currently an effective strategy to restore bee populations because of the highly specific circumstances under which those benefits would be observed. The current literature also does not provide adequate evidence in favor of this method due to the design and nature of the conducted studies.

\section{Methodology}

This paper reviews current literature on organic and conventional farming on bee populations from 33 articles and summarizes the findings as they apply to restoring bee populations. A review on agricultural trends in the United States will be provided alongside a summary of impacts of conventional farming on bees, including pesticides, monocropping and pollination practices. A literature review was selected over conducting a scientific study on bee populations because structuring and conducting a study of that nature exceeds the time limit available to complete the thesis and requires past experience studying migratory insects. It also does not synthesize the studies on the subject in a way that analyzes organic farming's overarching benefits, or risks, to bees. Articles used in this literature review were retrieved from Google Scholar and Portland State University Library catalogue database. Popular search terms used were: "agriculture," "organic," "conventional," "honey bee," "bumble bees" and "bees." Most of the cited literature was taken from a handful of specific journals: Journal of Applied Ecology, Ecology Letters, Science, Biological Conservation, PLoS One and Agriculture, Ecosystems and the Environment (Table 1). The USDA website also contributed to the findings of the paper for content on organic farming certification and practices. Bees were the focus of this paper because literature on bees and their decline is widespread and as such, the results of the research can be more easily translated to the public. There is more familiarity surrounding bees and their population trends which will help aid in the distribution of information and its acceptance. The thesis was structured as an in-depth literature review on organic and conventional agricultural practices and their effects on bees with a focus on the United States.

Table 1: Number of articles cited by journal, listed from largest to smallest.

\begin{tabular}{|l|l|}
\hline Journal Name & Number of Cited Articles (33) \\
\hline Journal of Applied Ecology & 5 \\
\hline Ecology Letters & 3 \\
\hline PLoS One & 3 \\
\hline Biological Conservation & 3 \\
\hline Science & 2 \\
\hline
\end{tabular}




\begin{tabular}{|l|l|}
\hline $\begin{array}{l}\text { Proceedings of the National Academy of } \\
\text { Sciences of the United States of America }\end{array}$ & 2 \\
\hline Agriculture, Ecosystems and the Environment & 2 \\
\hline Current Biology & 2 \\
\hline Journal of the Human Environment & 1 \\
\hline Trends in Ecology and Evolution & 1 \\
\hline Agronomy for Sustainable Development & 1 \\
\hline Synthesizing Ecology & 1 \\
\hline Ecological Applications & 1 \\
\hline The Year in Ecology \& Conservation Biology & 1 \\
\hline Insect Conservation & 1 \\
\hline Invertebrate Pathology & 1 \\
\hline Ecology and Evolution & 1 \\
\hline Diversity and Distributions & 1 \\
\hline Apidologie & 1 \\
\hline
\end{tabular}

Organic farming was used since it is a regulated program under the United States Department of Agriculture (USDA) where compliance to the guidelines are quantifiable and measurable on farms. Sustainable farming practices on the other hand, are undertaken in the absence of quantifiable conditions of operation, making potential benefits to bees more difficult to apply to a wider range of farms as conditions are inconsistent. Sustainable farming practices additionally cannot be compared between farms due to this reasoning. Organic farming benefits were grouped under three dominant effects after close analysis of the literature: increased floral resources, production of natural habitat and farm size. These topics will be addressed later in the "Benefits of Organic Agriculture" section. These three benefits capture the bulk of the generalized benefits of organic farming.

\section{Background}

This section of the paper provides a brief history of global population trends of bees, the "pollinator crisis," and the role of conventional agriculture in bee declines. The first observations of honey bee colony collapse have generated years of research aimed at explaining the declines of these much-needed organisms and constructing a global picture of pollinators in crisis. 
Pathogens and diseases are one such investigated explanation. Over the past 20 years in North America, 4 closely-related bumble bee species (Bombus) experienced a $96 \%$ reduction in abundance and range reductions up to $87 \%$ from their historic levels due to infection from the pathogen $N$. bombi (Cameron, 2010). Other causes of decline have been noted from a reduction in the number of people in the beekeeping profession. In Europe between 1985 and 2005, beekeeper populations declined by $31 \%$ resulting in a $25 \%$ reduction in overall honey bee colonies (Potts et al, 2010). On their own, factors such as disease and reduction in beekeepers are not responsible for explaining CCD. As colonies continued to experience decline, a broader scope of investigations took root to account for a variety of factors. The consensus among pollinator researchers now is that bee declines are attributable to a number of interacting factors at various scales. Land use change causes extensive habitat loss and fragmentation, making it one the most persistent and applicable factors contributing to bee declines as development and agricultural expansion continue. Other variables including reduced floral resources, climate change and pathogens amplify these effects, indicating that bee declines result from humanrelated actions regarding land management and bee keeping practices (Goulson et al, 2015; Potts et al, 2010). This research has solidified in the minds of the discourse community and general public that bee populations are indeed declining and the world is suffering from a "pollinator crisis."

Managed bee populations dominate the aforementioned research since their populations are easy to study when investigating multiple or singular factors related to their decline, subsequently, they exclude critical information regarding the status of wild pollinators. For CCD, this means that managed hives convey only a fraction of the story regarding bee populations. Information regarding wild bee populations is minimal compared to research on managed hives. Additionally, of the studies published on bee declines, most of them are isolated within North America or Europe, creating a bias in the discourse community (Goulson et al, 2015; Aizen et al, 2009; Ghazoul, 2005). Informational deficiencies from other countries also inhibit the ability to paint an accurate picture for bee population trends and thus give significantly more weight to the findings from North American and European studies. Data analyzed from the Food and Agricultural Organization (FAO) on global trends of managed beehives reveal a $1.5 \%$ increase per year and has lead to a total $45 \%$ increase of managed hives (Aizen et al, 2008). The data also reveal that factors associated with pollinator decline originate from a combined effect of elevated demands for pollinator dependent crops and an inability of available pollination services to meet those demands amidst a 23\% global increase in agricultural land use (Aizen et al, 2008; Ghazoul, 2005). The $45 \%$ increase in managed hives reported globally counters the losses reported in studies conducted in North America and Europe and generates uncertainty around the status of a "pollinator crisis." The lack of sufficient data on wild pollinators, a global perspective of both wild and managed bees, and a 45\% increased in managed hives undermines the argument that the world is suffering from a pollinator crisis (Aizen et al, 2009). Although globally managed populations of bee are increasing, both the United States and Europe are experiencing detrimental declines in their managed bee populations. Both wild and managed bees utilize farm 
resources and by analyzing the effects of organic farming practices, wild bees can be studied and benefits to both populations of bee can be achieved. The picture for wild bees again, is more unclear and their population dynamics cannot be stated with as much confidence. Although managed hive numbers appear to be consistently increasing, information on wild bee populations and conservation efforts are lacking, putting wild bees at risk (Winfree, 2010). These revelations however, do not discredit the threats and declines current human activity has manifested in both bee populations in North America and Europe and their potential to negatively affect other countries. It is an indicator that although one is not currently happening, a pollinator crisis may eventually emerge if current environmental management practices continue without regard to the preservation of critical pollinator species like bees.

A majority of the noted causes behind bee declines in managed colonies pertain to conventional agricultural practices and agricultural land use; monocropping and homogenization of landscapes has reduced diverse floral resources, land use change damages viable bee habitat and commercial (i.e. imported bees) colonies transport pests and diseases through exposure to a variety of chemicals and environments. Wild bees provide over $50 \%$ of the pollination services (Winfree et al, 2008) and their protection is vital to food production, ensuring food security and sustaining the health and productivity of natural ecosystems. Unlike managed colonies, wild bees depend on both habitat and food resources from their environment for survival. Managed bees need depend on food resources only since their hives are provided by their beekeepers. Both nesting and food resources disappear in the wake of conventional agricultural practices. Organic farming provides an agricultural management alternative that has the potential to counteract the adverse effects of conventional farming on both wild and managed bee populations by providing more floral resources, habitat and elevated biodiversity. The USDA Organic Certification is delegated to farms abstaining from synthetic chemicals to fertilize or combat pests/weeds, establishing buffers for natural habitat surrounding the farm and performing intercropping and crop rotation (USDA, 2016). The absence of harmful pesticides, the addition of natural areas for bees and the production of more diverse crops directly targets multiple factors connected to declines in bee populations. Agricultural land use dominates global land types and utilizing it to revive and protect bees is a plausible solution to restore colony numbers in the United States and prevent a future "pollinator crisis." Not only does it allow managed and wild bees to benefit, research on its efficacy can fill in the missing gaps in knowledge on wild bee populations.

\section{Current Trends in United States Agriculture and Dependence on Bees}

This section of the paper summarizes recent trends and the scope of agriculture in the United States for both conventional and organic acreage. The United States is a hub of agricultural 


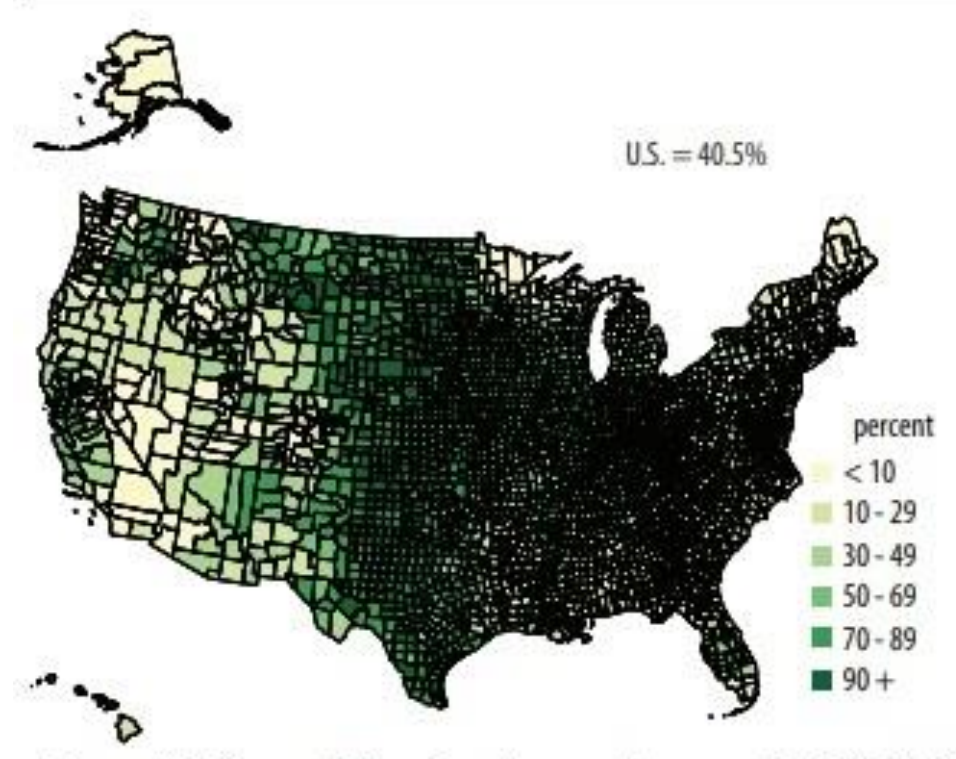

Figure 1: Percent farmland cover by county (USDA, 2012)

Source: USDA MASS, 2012 Census of Agriculture.

activity that continues to change, taking with it all the various approaches of agricultural operation. In 2014, the USDA NASS highlight on farms and farmland reported that agricultural land use in the United States currently occupies 915 million acres of land (USDA, 2014). This is a decline from 922 million acres in the previous year's report. Of the 915 million total acres, approximately 390 million are utilized for crop production. Organic farmland occupies 50 million acres and has seen 20\% and 11\% increases in the years 2014-2015 and 2015-2016, respectively (Figure 2) (USDA, 2017). Despite the decline in farm acreage across the country, the average farm size is increasing from 418 acres to 434 but with a concurrent decline in the number of farms (Figure 3) (USDA, 2014). This trend indicates farms in the United States are becoming larger but fewer in number, a trademark characteristic of large-scale conventional farm management. It may also translate to established organic farms expanding and absorbing more land. The overarching trend present here is an increase on the intensity of farms with a decreasing concentration of farms (i.e. a single farm possess more agricultural weight/impact in a system than in previous years). With more impact being exuded onto surrounding systems, the mitigation provided by organic farms becomes more crucial to the health of bees and the environment. 
Million acres

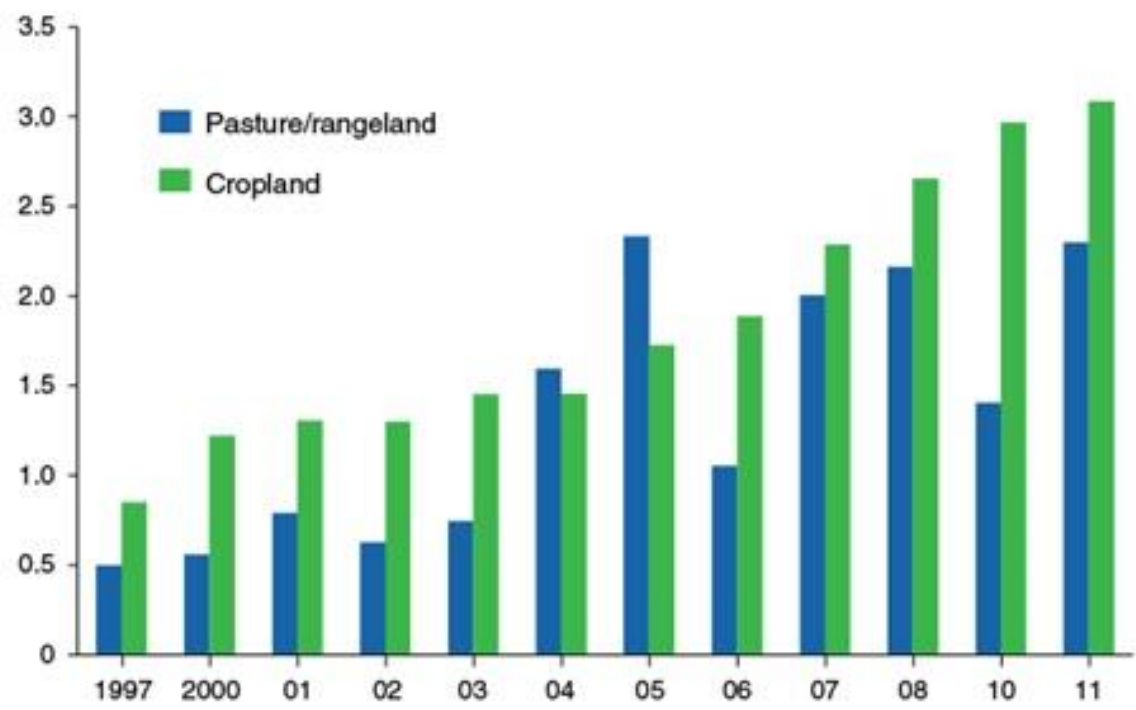

Source: USDA, Economic Research Service, based on information from USDA-accredited State and private organic certifiers.

-On November 22, 2013, data underlying the chart "Certfled organic cropland and pasture reached nearly 5.4 million acres in 2011" was corrected. The correct number of certfied organic pasture/rangeland acres in 2005 is 2.331 million. The correct number of certified organic pasture/rangeland acres in 2010 is 1.405 million; the correct number of certifled organic cropland acres in 2010 is 2966 million.

Figure 2: Organic cropland and pastureland in millions of acres from 1997-2011(USDA, 2017)

The United States has a dearth of substantial data on the dependency of managed, wild or commercially imported bees on the agriculture industry. Without an understanding of the populations of bee depended upon in pollination, targeting effective, mitigating organic farming practices for bees of any species will be difficult and contain uncertainty. A majority of the reports are economic and represent bee pollination services in USD (\$) by crop yield. While this is useful for business analysis by farmers and government officials, it does not provide adequate information for the quantity or type of bees responsible for those figures. More research is needed to investigate the ratio of wild, managed and commercially-shipped bees surrounding pollination services in United States agriculture. Bee visitation data and more careful tracking on the number of commercially imported bees are both possible solutions. Through the current available literature on wild bee pollination and trends in managed hives however, it can be 


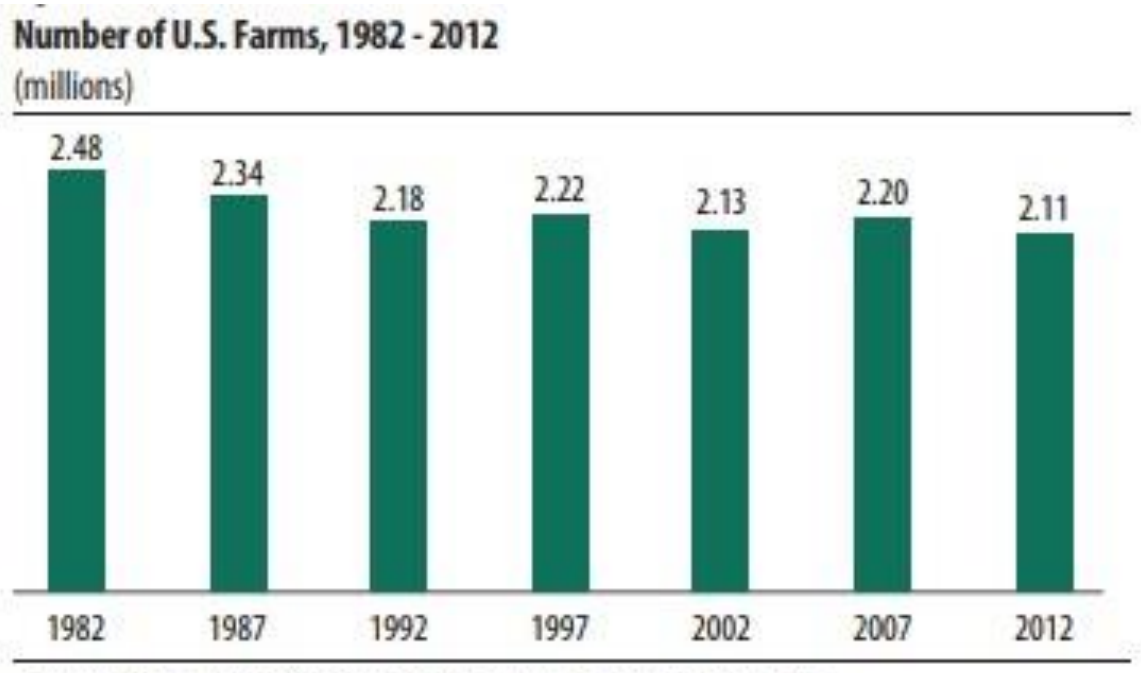

Source: USOA MASS, 2012 Census of Agriculture and eorlier census dato.

Figure 3: Number of farms in millions in the United States from 1982-2012 (USDA, 2014)

inferred that wild bees may soon provide the majority of pollination services. North America experienced a $49.5 \%$ decline in managed honey bee colonies and the number of honey-producing colonies fell from 5.9 million to 2.3 million by 2008; a decline of $61 \%$ (vanEngeldorp et al, 2010). Wild bee populations have been found to provide all pollination services for farms and can handle the capacity demanded by pollination-heavy crops, provided that the communities have viable habitat nearby (Kremen et al, 2002). Since wild bees have the capacity to take on the duties of commercially imported and managed bees, this indicates that wild pollinators likely contribute a greater share of pollination services to agriculture. The exact quantification of these services however, have not been determined. This also indicates that although wild bee pollination services have a large capacity in their usefulness to agriculture, farmers rely on the services of managed and commercial hives more. Fostering healthier wild bee populations through organic farming may reduce dependency on managed hives. In turn, this may make them healthier as well since the distribution of labor is more balanced. This does not mean that wild bees are more worthy of protection and restoration efforts than managed bees but it does emphasize the importance organic farms have in providing natural habitat for bees and restoring natural services.

\section{Conventional Agriculture's Role in Bee Population Decline}

Conventional, or industrial, agriculture has been associated over time by a handful of key practices: aerial and/or terrestrial application of pesticides, monocropping (i.e. growing and harvesting a single crop variety such as corn), growing crops on the same plot of land multiple seasons in a row, planting genetically modified seeds, tilling and the combined application of all of these practices over a large area of land. The goals of conventional agriculture revolve around 
maximizing yields and making a profit by reducing costs, which explains the need for large areas, pesticides, fertilizers and exhaustive use of the of land and its resources. Across the world today, large swaths of land are being cleared for intensive agriculture practices, notably the Amazon rainforest. Widespread clearing of land in this manner for conventional agriculture removes habitat and resources for bees and other organisms which then face negative externalities of the agricultural industry.

\section{Pesticides}

Pesticide application is one of the most studied topics in agriculture and its impact on bees is widespread. In recent years, restrictions on chemicals and application techniques has limited conventional farmers in their pest-fighting options and has thus limited the direct exposure of bees to harmful chemicals. Despite careful regulations of toxic chemical compounds, it does not entirely control their release into the environment and or reduce the likelihood of the compounds coming into contact with bees. Neonicotinoids, among other toxic compounds, can be found in plant pollen and nectar where it adheres to bees' legs and bodies during foraging in the spring and summer (Krupke et al, 2010). These contaminated vectors are capable of transporting sickness into hives and increasing bee mortality. Pollen is often fed to juvenile and larval bees (the brood) and if the pollen contains toxins, the population dynamic of the colony/or community will shift from the increased mortalities among the younger group of bees. Bee colonies or communities facing population declines are less likely survive in the face of environmental stressors and can lead to CCD or loss of the community. The same compounds can be found at parts per million ( $\mathrm{ppm}$ ) and parts per billion (ppb) concentrations in hives, bee products and bees themselves that cause lethal effects (Johnson et al, 2010). While more stringent rules on pesticides can limit direct exposure to bees, indirect exposure is still a prevalent issue that maintains ties to reduced bee colony fitness and survival.

\section{Monocropping}

Monocropping in conventional agriculture removes basic necessities needed by any organism for survival: food and available habitat. For bees, homogenization of vegetation reduces floral resources and the clearing of land destroys their habitat. Reduced availability of habitat and nutritional resources are stressors for any population and limit organisms from surviving for prolonged periods of time. In multiple studies conducted on conventional agricultural practices, the combined absence of habitat for bees and their floral resources have indicated a reduction in species diversity, abundance and richness (Le Feon, 2010; Gabriel et al, 2013: Goulson et al, 2018). Gabriel et al, 2013 takes a different perspective by investigating landscape productivity through crop yields and bee species density where higher crop yields produce a negative relationship with bee density, indicating that large, highly productive farms reduce the number of bee species per area. Conventional farms operating on the demand for large yields are not seen as valuable resource reservoirs by bees and cause reduced visitation and pollination as they are drawn to other, more attractive resource pools. This forces bees in resource "deserts" to either 
migrate or increase their foraging range, both of which are stressful adaptations, or risk death. It should also be noted that landscapes converted into monocropping operations undergo fairly rapid change, leaving little time for populations to successfully adapt and there is little effort to offset these direct alterations to the landscape. Monocropping causes two of the most noted detrimental impacts to bees by removing the resources necessary for basic survival. Without the support by the most basic needs, bee populations living in conventional agricultural landscapes face serious threats.

\section{Pollination Practices}

Conventional agriculture depends heavily on imported and managed bee hives for the pollination of crops due to high yield demands, but these populations of bee present threats to native, wild populations as well as to their own colonies. These bees are exposed to multiple environments in both the United States and other countries and are also kept in close quarters. Both conditions are ingredients necessary for breeding sickness. Managed colonies within the United States pose more of a risk for contracting disease and pests but the application of non-native bee colonies shipped from other regions of the globe carry diseases and pests as well (Goulson et al, 2018; Potts et al, 2010). Many of the studies covering overarching impacts on bee colonies address the interaction of the aforementioned stressors but clearly state that minimal research is present to assess interacting stressors on actual bee populations. Diseases however, provide a glimpse into these interacting factors. A common pathogen afflicting native bumble bees in the United States is Crithidia bombi or C. bombi. This pathogen is carried from non-native, commercial bumble bee hives onto flowers used by native bee communities and can infect $20 \%$ of native, wild species within the first three months of exposure, and by 18 weeks can reach nearly $100 \%$ infection (Otterstatter \& Thomson, 2008). Pathogens like C. Bombi are often transmitted via direct contact with infected individuals or pollen, which are both highly active vectors for bee species. Organic farms in this manner, come across as an appealing solution for the restoration of native, wild bee pollinators. In this hypothesis, wild bee presence and population growth provided by natural areas on organic farms is seen as a way to reduce dependency on commercial hives and reduce risk of infection of the regenerating populations since more wild bees are providing natural pollination services. Conventional agriculture presents multiple negative effects on bee populations both wild and managed and emphasizes the need to reassess the United States' approach to agriculture if healthy bee populations are to continue to survive. Organic agriculture addresses these problems by providing more floral resources, increased natural habitat and altering farm size.

\section{Benefits of Organic Agriculture on Bees}

Three categories of benefits to address the problems presented by conventional farming practices have emerged in the literature: increasing floral resources to combat monocropping, providing more natural habitat to counteract large, unbroken farmland, and altering the size of the farm to 
mitigate homogenization and landscape-scale effects. All these categories possess overlap, particularly between natural habitat and increased floral resources. Naturally, more habitat area will increase the amount of food available for survival and the more food resources there are, the more elements are being contributed to make a habitat. It is critical to note that the majority of research has been conducted on the general richness, biodiversity and abundance of bee species and the lens studies typically use to analyze those factors is through natural habitat. Another important note is the studies that investigate comparisons between conventional and organic agriculture do not specify the practices being conducted on the organic farms or target a specific practice in their research. These problems arising in the literature present impediments when attempting to identify useful practices of organic farming and how bee communities respond. Specific practices conversely, cannot be stated to have any more or less impact on bee populations than another. It should also be noted that the articles cited do not distinguish between managed and wild bees but instead focus on different species of bees (i.e. honey bees and bumblebees) and many generalize bee species and populations (e.g. "solitary bees" in comparison to "bumblebees"). Some articles specify wild bees in their results but those articles are limited in the application of their findings since other wild pollinators included in the study. This reduces the validity of applying noted findings and practices to bees for the purpose of aiding bee populations. While the literature reviewed in this section builds a strong case for the benefits of organic compared to conventional farming, evidence supporting the application of organic farming as a method to combat declines in bee populations is minimal and requires specific conditions for significant improvements.

\section{Increased Floral Resources}

Intercropping, planting diversified crops and establishing buffer zones on organic farms diversify and expand floral and food resources for bees. Whether wild or managed bee populations are making use of a farm, both require the provided floral resources to bolster their populations. Within a few years of converting to organic practices, increased availability of food for bees improved overall pollination success (Andersson, 2012). This indicates a steadily increasing bee population and abundance since the time of conversion and holds potential for future stability if those same practices continue. If populations of wild bees remain steady and continue to rise, the farmers may also benefit in their ability to increase crop yields in the presence of more wild pollinators. Increasing floral resources reveals the effectiveness of changing crop cultivation practices on improving the health of bee populations and its immediacy. It does not however, provide any suggestion to the long-term benefits if the elevated presence of bees is maintained or the specific crop practices used to attain this result. Despite the benefits provided to bees by switching cultivation techniques, studies also lack specificity in that they analyze cultivation as a whole unit rather than investigating specific methods used to attain these results.

Organic farming prevents chemical elimination of weeds and other plant-based pests but the presence of vegetation outside crops provides additional floral resources such as red clover blossoms. Between organic, conventional and genetically modified (GM) crop farms, pollination 
capacity by bees was fully reached on organic farms with a total of 342 bees, midway on conventional farms with 230 bees and low on GM farms with just 101 bees (Morandin \& Winston, 2005). The increased bee abundance is predicted to be responsible for the increased pollination capacity provided by the variety of crops present. A relatively unexplored avenue of the benefits of organic farming is the benefit of weeds to bees and other pollinators. In a unique study, Nicholls and Altieri (2013), argue that increasing plant biodiversity on farms through proper weed management practices provide favorable and stable habitats for bees that will subsequently improve pollination services for wild pollinator species. Weeds provide diverse food and nesting, reduce homogenization and fragmentation of the landscape while also reducing bee pests. Reduced disturbance responsible for hindering establishment of pollinator populations can additionally be achieved through weeds by connecting habitats and ecosystems. Because organic farming prohibits the use of pesticides and is known for diversified crop production and natural buffers, weed management to improve bee populations would likely improve floral resources and provide more habitat for many different species of bee. More research is needed to investigate the benefits of this particular practice of organic farming, however, before its potential application is cited to significantly benefit bee communities.

Increasing the amount of floral resources through organic crop management is very effective at increasing the abundance of bees at minimum and may help bees resist further declines in their populations. The articles are limiting, however, in their ability to correlate increased abundance to beneficial changes in bee population dynamics since studies lack long-term monitoring. It cannot be assumed that floral resources alone will impact the bees' ability to restore their populations. An increase in floral resources on an organic farm may cause increases in abundance and diversity from attracting a larger ratio of bees in comparison to the total landscape without altering their overall population dynamic. Other questions require answers as well. How much more diverse does an organic farm have to be in order to produce an increased level of abundance? In comparison to other organic practices, how much more does increased food diversity impact bees? Will the increased abundance trigger negative competition effects among bees and other pollinators in the organic farm ecosystem? Current literature does not address these questions and as a result, organic farming loses traction in its power to benefit bees through intercropping, natural buffers and diversified vegetation.

\section{Natural Habitat}

Providing habitat for organisms is a foundational practice in conservation and restoration efforts that is often met with success. On organic farms, the presence of buffer zones between fields and the restoration of native habitat surrounding the farm provides increased floral resources and nesting sites simultaneously. The bees' ability to nest closer to their food source reduces foraging risk and enables colonies to have access to a greater amount of nutritional resources for population growth. The energy directed to prolonged foraging can now be diverted to reproduction and growth. Competition for other viable habitat is also reduced and causes a reduction in overall stress on bee species or communities. A 5-20\% increase in organic farming 
at a landscape scale increased the richness of bee species overall by $50 \%$ where solitary bees saw an increase of $60 \%$ and $105 \%$ for bumble bees. These effects were strongest from a 250-500-m radius from 21 organic fallows strips. These effects were also noted to take place at local and landscape scales (Hulzschuh et al, 2007). Bumble bees likely respond more favorably to organic farming than solitary bees because solitary bees are able to travel farther and access more food and habitat resources and do not live in large hives. Thus, bees that live in hives might benefit more from organic farming practices than solitary pollinators. This does have its drawbacks however.

One drawback of natural habitat on organic farms is the disconnect from the surrounding landscape. Edges of fields and farms contain the greatest level of diversity, but in order to maximize the effects of organic farming, management must also be done in the area surrounding the farm (Gabriel et al, 2010). Organic farms alone cannot achieve the habitat requirements necessary to successfully restore bee health and populations; efforts will also need to be taken by other types of landowners to broaden connectivity. Isolated populations of organisms are known to suffer from stagnated growth due to the limited amount of genetic resources and smaller populations are less able to defend themselves against natural environmental stressors. Organic farms have other demands to meet as a business such as making profits and growing marketable crops. Conflicting interests and needs of farms limit their ability to provide adequate natural habitat and as such, the provided benefits of buffers and vegetation restoration has its limitations despite their noted benefits.

Depending on the habitat requirements of the bee, not all bee species will respond the same to natural habitat restoration. Seventy percent of native bees in the United States nest underground and the European honey bee, a non-native but long-established species, nests in the hollows of trees (USDA, 2011). Cover crops and natural buffer zones on farms, therefore, are essential for maintaining healthy populations of bees by reducing the amount of vulnerable bare ground and providing trees for honey bee colonies. Bee species increase in richness and abundance by $30 \%$ and 50\%, respectively, but is varied between landscape and organism type (Bengtsson, 2005). The benefits of organic farming at smaller scales reflect individual behaviors while the large scale will reflect population dynamics. This indicates organic farming practices can be utilized to benefit solitary or social bee pollinators based on the scale of implementation but cannot extend enough to instigate population-level changes.

Farmland and the surrounding landscape interact with bee communities in such a way that organic farming benefits are augmented when practiced in more homogenous landscapes. Regions where the landscape is more heterogeneous (i.e. more diverse land types) would not benefit as much from organic farming practices, however since regions where agriculture is widespread, organic farms would act as oases for bees and other species in a more homogenous landscape. As such, bee populations living near homogenous agricultural landscapes would be able to benefit from organic farm practices being implemented. These benefits (e.g. increased species richness and diversity), however, are likely going to be confined to agriculture-intensive landscapes and exclude heterogeneous regions or regions with prevalent organic practices 
(Bengtsson, 2005). One study found an average of 5.33 species of bee on both conventional and organic farms in homogeneous landscapes but the number declines when considering the high average number of bee species on organic farms alone: 7.67 (Rundolf et al, 2008). Despite efforts to implement organic practices, it is important to also recognize the history and current state of the land when considering actions to alter it. Organic farming may not be as useful as previously thought if practices are put into place in heterogeneous landscapes where buffers between natural and managed land are dense. An increase in heterogeneity can cause a decline in bee species' richness and abundance in areas with historically dense buffer zones with the exception of bumble bees. Bumble bees favor increased numbers of patches in the landscape mosaic (Aguirre-Gutirrez et al, 2015). This will limit the number of regions capable of implementing successfully beneficial organic farming practices, and the species of bee, if the landscape history does not favor increased heterogeneity. Switching to organic farming practices also includes modification of the landscape to promote natural habitat resources. Habitat corridors and buffer development in the sense of landscape management provide significant benefits to bees without changing agricultural practices. In a healthy landscape mosaic, the establishment of conservation land for bee species would increase their richness, abundance and diversity in both the landscape and on farm fields. Lastly, natural habitat diversity is more responsible for increased levels of pollinators than farming practices (i.e. more local and landscape scale effects) (Chateil et al, 2015). Despite these findings that reduce organic farming's effectiveness in regions with a more heterogeneous landscape, it leaves opportunity open for dramatic benefits in regions of widespread agricultural land use.

Literature on natural habitat restoration on organic farms has revealed that this practice generates some of the largest noted benefits to bees but with significant drawbacks. Despite the positive effects on abundance, diversity and richness, the benefits lose their significance in heterogeneous landscapes and are in conflict with competing needs of farms. Organic farms are often present in areas of the United States that are already taking heavy action to restore habitats of various organisms or have a smaller percentage of intensive agricultural land (e.g. the Pacific Northwest). The midwestern United States, on the other hand, has widespread conventional land 


\section{Fig. 1. Number of Certified Organic Farms by State, 2016}

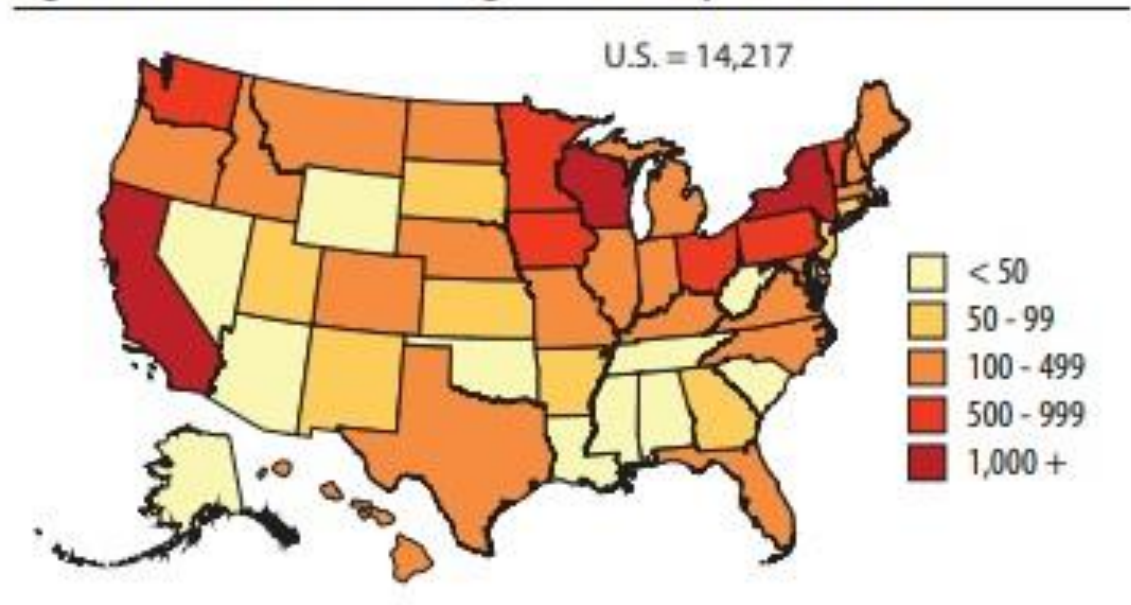

Figure 4: Number of certified Organic farms in the United States by state (USDA, 2016)

use and thus, a higher potential to reap benefits of organic farming but is not an area with high organic activity (Figure 4). It also lacks evidence on temporal changes in bee populations as many studies investigate individual bee numbers on farms alone of focus on diversity of bee species. Population dynamics include information on reproductive patterns, nesting behavior and migration. Analyzing long-term nesting behaviors of bees in response to agricultural practices may provide a different perspective on the effects of organic farming that are not being observed by studying bee visitation to farms and focuses on wild bees, a currently deficient area of research. Different strategies will also need to be developed for regions with heterogeneous landscapes. Developing productive and supportive mosaic landscapes may be the best approach in these regions to work with the diversity and natural areas already present. This will ensure the ecosystem as a whole is healthy and sustainable with little or no human intervention.

\section{Farm Size}

The debate of whether species benefit more from "many small or one large" conservation area(s) has been a topic of discussion within conservation practice since its development. The varying size of farms presents a similar question when considering the impact of organic farming on bees in agriculturally-dominant landscapes. Most conventional farms occupy a single, large, unbroken region of land containing a single crop. Devoid of adequate habitat and food resources, large agricultural operations are a desert for bee communities. By dividing agriculturally-dominant landscapes into multiple smaller farms, natural buffers containing diverse vegetation and habitat can provide previously absent food and nesting resources. The benefits of these actions could be bolstered by converting farms to organic practices, creating a synergistic effect. When comparing small and large conventional and organic farms, both small scale and organic farms benefitted bees; however organic farms provide increased bee species abundance and generate more floral 
resources, creating a positive feedback loop (Happe, 2018). The smaller sized farms benefit bees with shorter travel ranges and contain many of the necessary resources for survival within a close distance. Farm size reduction will naturally reduce crop yields produced on the farmland. By reducing the farm size, and subsequently the yields, bee biodiversity increases. Reduced crop yields on organic farms increase individual bumble bee numbers by $23 \%$, bumble bee species by $27 \%$, solitary bee individuals by $26 \%$ and solitary species by $21 \%$ (Gabriel et al, 2013). In this sense, reductions in farm size produce beneficial indirect effects for bees that could extend beyond reduction in crop yields. Findings for the benefits of smaller, organic farms however, is inconsistent and produce uncertainty in concluding their benefits.

Even if small farms are able to generate increased bee activity and abundance, it may not be enough to produce significant beneficial, long-term impacts on populations of bees. Identifying long-term benefits is critical to the reversal of bee declines and their restoration. Despite smaller, organic farms containing higher numbers of bees ( $405 \%$ more bees), the differences between large, conventional farms are not significant (Belfrage et al, 2005). Although more bees were present, the difference is not significant enough to warrant the conversion to organic farming specifically for the benefits provided by reduction in size. A promising insight in this study however, is that when there were more birds present the number of pollinators, including bees, was also greater (Belfrage et al, 2005). Birds could thus be utilized as "pioneer pollinators" for bees and encourage restoration by paving pathways for bees to reach habitat and food resources. They may also serve as indicator species for bees, although more research would have to be conducted on which species of bird indicate the presence or absence of certain species of bee.

The debate of "many small or one large" appears, in some instances, to favor bees when smaller farms are present but does not sufficiently address the effect of quantity. Evidence from the previous sections suggests that multiple organic farms will not significantly improve bee communities due to the heterogeneity of the landscape that organic farms inherently create. Another important aspect to consider is temporal trends in bee communities throughout the conversion from conventional to organic or vise versa. Disturbance and recovery of the environment at various stages in conversion will impact observed patterns of bees. Many of these studies compare well-established conventional and organic farms and their size differences, preventing long-term, temporal trends from being uncovered. Farm size is, consequently, an inconclusive sector in organic practices pertaining to the benefits of bee populations. The lack of consensus among research on this topic makes it near-impossible to confidently state the potential of reduced farm size on bees. Long-term studies on this topic are also lacking. Evidently, specifications on more types of bees and their long-term response to reduction in farm size would be useful in determining which bees would benefit from many, smaller organic farms.

\section{Conclusion}


Declines in bee populations in the United States are in large part attributed to conventional agricultural practices. Both wild and managed bees face environmental stress from reduced habitat, food and toxins from intensified food production nationwide. The pollination and natural services provided by bees is crucial for continued food production and vibrant, productive ecosystems but those may be lost if current agricultural practices continue down their current path. Organic farming practices through the USDA Organic Certification acknowledge the environmental risk and degradation brought about by conventional farming and take action to mitigate them. Of the requirements needed for organic certification, literature has focused on floral resource abundance, natural habitat and farm size as the primary contributing factors influencing bee species and populations.

In summary, increasing floral resources on organic farms does attract a greater diversity and abundance of bees and improves pollination success but does not provide any inclination to the long-term benefits of this practice. By studying organic farms in isolation, studies also ignore the possibility that more food availability on organic farms may be drawing a larger number of bees away from other regions of the landscape without changing the population dynamic. Natural habitat in noted to produce multiple benefits to bees but is highly limited to homogeneous landscapes to generate significant impacts. Smaller farms reduce yields which provides benefits to bees but farm size does not present any information about the effect of quantity in regards to the debate of "many small or single large" conservation areas.

Multiple studies limit the scope to general bee diversity, abundance and richness as it pertains to visitation on farms. Increased diversity, abundance or richness is not indicative of elevated populations of bees but is the primary focus of multiple studies investigating the effects of organic farming on bees. Focusing future research on nesting and reproductive behavior surrounding changes to agricultural practices may provide new insights to bee populations not currently evident by studying visitation to farms. Longitudinal studies on bees and pollinators with respect to organic farming focus on the total abundance and richness of species but have little focus on studying communities or populations of bees. Without this information it is unknown whether or not organic farming practices generate an environment where bees are able to recover their numbers. More research needs to investigate known populations of bees living near recently converted organic farms and assess their population dynamics over time. Another deficient area of research is on the population trends of wild bees, specifically. Some studies do not distinguish between the species of bees being studied.

Additionally, increased biodiversity in farm ecosystems may not give bee communities the space to recover their population if niche space is highly competitive between other pollinators. This paper showed that the direction of pollinator recovery and conservation may be better directed at landscape scale recovery in agriculturally-intensive regions. This will provide more natural habitat for wild bee pollinators and alleviate dependency on managed hives that may pose risks to wild populations from disease. These articles also only provide short-term analyses of the benefits of organic farming and lack assessments of population dynamics. Very few models exist in the current literature that make projections about how certain farming practices will impact 
pollinators as a group through time. Current information on the efficacy of general conservation and recovery tactics for bee species might be a useful resource in pinpointing strategies to introduce to farms and around agricultural regions, regardless of its organic certification status. Since organic farming certification and its practices are thus far limited in their potential to restore bees, this would provide an alternative to reduce the burden agriculture has on bees in general.

This paper is limited in the number of studies reviewed (33) due to time constraints. It also does not address nesting behavior of wild and managed bees in regards to farm practices which are critical to understanding changes in community numbers and populations. An analytical review of numerical results from a larger number of studies would additionally provide a better understanding of types of the types and quantity of vegetation used, the exact farm size to generate changes in bee populations and other, similar data pertaining to this topic. This paper also does not review enough studies to be able to specify which organic practices are responsible for producing the results outlined throughout the review. This review would have benefitted from a larger number of paper reviews, an analytical component detailing specific farm practices, bee species, vegetation/crop types and a comparison between which practices produce what results to what degree. Additional research can focus on investigating nesting and reproductive trends of wild bee species under conversion to organic farming over multiple seasons. Pollinators also utilize personal gardens and flower beds for food and nesting. Gardening is a popular pastime in the United States that almost anyone can participate in. Gardens exist in agricultural landscapes but also in urbanized regions and the hobby encompasses a diverse range of people (e.g. urban gardeners, families beautifying their yards). Gardening and flower beds are also being used to mitigate bee declines across the United States. Targeting and studying gardening practices to benefit bees could be another avenue to investigate since there is a reduced conflict of interest compared to farming and the effect of homogeneity may not be present due to the widespread nature of the hobby. Another practical approach for future research is weighing the benefits of conservation/restoration efforts for bees against the benefits of organic farming to parse out any beneficial conclusions. If organic farming outweighs standard conservation/restoration efforts under the same circumstances, more effort should be directed at farm conversion. The combined effect of the two may also produce greater results than either one alone. Lastly, sustainable farming is not governed by a federal agency or guided by requirements but many farmers adopt sustainable practices to benefits the environment. Agroforestry and using farm animals integrated with crops are two examples.

In conclusion, the benefits of organic farming to bees are limited and depend on specific contexts in order to produce benefits. Even if those benefits do exist, there is little indication that those effects will result in a change in population dynamics and reversal of bee declines in both wild and managed species. There are still multiple questions that need answering: What are the potential overlaps between sustainable and organic farming? Could sustainable practices benefit bee populations in ways USDA organic farming cannot? Although organic farming was not revealed to significantly mitigate bee declines in this literature review, further efforts to 
investigate how conventional agriculture can be altered or re-developed to mitigate its effects on bees is needed. Current practices threaten the continued existence of wild and managed bees and without some mitigation or reform, the "pollinator crisis" may become a global reality.

Cited Literature

Aguirre-Gutierrez, J et al (2015). Susceptibility of pollinators to ongoing landscape changes depends on landscape history. Diversity and Distributions, 21:1129-1140.

Aizen, M \& Harder, L. (2009). The global stock of domesticated honey bees is growing slower than agricultural demand for pollination, Current Biology, 19(11). Pp. 915-918.

Aizen et al (2008). Long term global trends in Crop yield and production reveal no current pollination shortage but increasing pollinator dependency. Current Biology, 18(20). pp $1572-1587$

Anderson et al (2012). Organic farming improves pollination success in strawberries. PLoS One, 7(2).

Belfrage et al (2005). The effects of farm size and organic farming on diversity of birds, pollinators and plants in a Swedish landscape. Journal of the Human Environment, 34(8). 582588.

Bengtsson et al (2005). The effects of organic agriculture on biodiversity and abundance: a meta-analysis. Journal of Applied Ecology, 42, 261-269.

Benjamin et al (2014). Pollinator body size mediates the scale at which land use drives crop pollination services. Journal of Applied Ecology, 51. 440-449.

Brittain et al (2010). Organic farming in isolated landscapes does not benefit flowervisiting insects and pollination. Biological Conservation, 143(8). 1860-1867.

Cameron et al (2010). Patterns of widespread decline in North American bumble bees, PNAS, 108(2). Pp 662-667

Gabriel et al (2013). Food production vs. biodiversity:comparing organic and conventional agriculture. Journal of Applied Ecology, 50: 355-364. 
Gabriel et al (2010). Scale matters: the impact of organic farming on biodiversity at different spatial scales. Ecology Letters, 13: 858-869.

Galindo, G. Rickard, L. Rao, S. (n.d.) Guide to Common Pollinators in the Willamette Valley in Western Oregon. Retrieved from Oregon State University website: https://static1.squarespace.com/static/5a849d4c8dd041c9c07a8e4c/t/5aa5f494e4966bde3669b34 4/1520825503142/rao-may-2016-edited-pasture-tri-fold-bee-guide.pdf

Garibaldi et al (2013). Wild pollinators enhance fruit set of crops regardless of honey bee abundance. Science, 340(6127).

Ghazoul, J. (2005). Buzziness as usual? Questioning the global pollination crisis. Ecology and Evolution, 20(7). 367-373.

Goulson, D. et al (2015). Bee declines driven by combined stress from parasites, pesticides and lack of flowers. Science, 347(6229).

Happe et al (2018). Small scale agricultural landscapes and organic management support wild bee communities of cereal field boundaries. Agriculture, Ecosystems and the Environment, 254: 92-98.

Holzschuh, A., Steffan-Dewenter, I. \& Tscharntke, T. (2007). Agricultural landscapes with organic crops support higher pollinator diversity. Synthesizing Ecology, 117(3). 354-361.

Isaacs \& Kirk (2010). Pollination services provided to small and large highbush blueberry fields by wild and managed bees. Journal of Applied Ecology, 47, 841-849.

Johnson et al (2010). Pesticides and honey bee toxicity. Apidologie, 41, 312-331.

Kennedy et al (2013). A global quantitative synthesis of local and landscape effects on wild bee pollinators in agroecosystems. Ecology Letters, 16(5).

Kremen et al (2002). Crop pollination from native bees at risk from agricultural intensification. PNAS, 99(26), 16812-16816.

Kremen et al (2004). The area requirements of an ecosystem service: crop pollination by native bee communities in California. Ecology Letters, 7: 1109-1119. 
Krupke et al (2012). Multiple Routes of Pesticide Exposure for Honey Bees Living Near Agricultural Fields. PLoS One, 7(1).

Le Feon et al (2010). Intensification of Agriculture, landscape composition and wild bee communities: a large scale study in four European countries. Agriculture, Ecosystems and the Environment, 137. 143-150

Lentini et al (2012). Supporting wild pollinators in a temperate agricultural landscape; Maintaining mosaics of natural features and production. Biological Conservation, 149: 84-92.

Morandin, L. \& Winston, M. L. (2005). Wild bee abundance and seed production in conventional, organic and genetically modified canola. Ecological Applications, 15(3). 871-881.

Nicholls \& Altieri. Plant biodiversity enhances bees and other insect pollinators in agroecosystems. A review. Agronomy for Sustainable Development, Springer Verlag/EDP Sciences/INRA, 2013, 33 (2), pp.257-274.

Otterstatter, M. C. \& Thomson, J. D. (2008). Does pathogen spillover from commercially reared bumblebees threaten wild pollinators? PLOS ONE, 3(7).

Pereira et al (2015). The management of bee communities by intercropping with flowering basil (Ocimum basilicum) enhances pollination and yield of bell pepper (Capsicum annuum). Insect Conservation, 19: 479-486.

Potts et al (2010). Global Pollinator Declines: Trends, Impacts and Drivers, Trends in Ecology and Evolution, 25(6). pp. 345-353

Rundolf, M., Nilsson, H. \& Smith, H. (2007). Interacting effects of farming practice and landscape context on bumble bees. Biological Conservation, 141: 417-426.

United States Department of Agriculture (2017). 2016 Certified Organic Survey (20176). Retrieved from https://www.nass.usda.gov/Publications/Highlights/2017/2016_Certified_Organic_Survey_Highl ights.pdf

United States Department of Agriculture, Catherine Greene (2013). Growth Patterns in the U.S. Organic Industry. Retrieved from https://www.ers.usda.gov/amberwaves/2013/october/growth-patterns-in-the-us-organic-industry/ 
United States Department of Agriculture (2014). Farms and Farmland (ACH12-13).

Retrieved from

https://www.nass.usda.gov/Publications/Highlights/2014/Highlights_Farms_and_Farmland.pdf

USDA Forest Service, Pollinator Partnership (2011). Bee Basics: An Introduction to Our Native Bees. USDA, Pollinator Partnership.

vanEngelsdorp \& Meixner (2009). A historical review of managed honey bee populations in Europe and the United States and the factors that may affect them. Journal of Invertebrate Pathology, 103, 80-95.

Winfree, R. (2010). The conservation and restoration of wild bees. The Year in Ecology and Conservation Biology, 11(95). 169-197.

Winfree et al (2008). Wild bee pollinators provide the majority of visitation across landuse gradients in New Jersey and Pennsylvania, USA. Journal of Applied Ecology, 45: 793-802. 